\title{
Traceability and vertical co-ordination in the Italian dairy chain: A transaction cost approach
}

\author{
A. Banterle, S. Stranieri and L. Baldi ${ }^{1}$ \\ Department of Agricultural, Food and Environmental Economics, University of Milan, Via Celoria 2, 20133 Milano, Italy, \\ alessandro.banterle@unimi.it
}

\begin{abstract}
The purpose of this paper is to analyse how the introduction of a voluntary traceability system affects the organisation of economic relationships throughout the Italian dairy chain. Using the theoretical framework of transaction cost economics, we assess whether traceability increases the degree of vertical co-ordination and changes the level of the transaction key features, i.e. degree of asset specificity, uncertainty and frequency of transactions. A survey was conducted by questionnaire to assess changes in vertical relations, and to underline the different organisational solutions of dairy firms we carried out factor and cluster analysis. The results show increased bilateral dependency among the economic agents as a consequence of the rise in human, physical and site assets. At the same time, growth in the frequency and quantity of information exchanged is observed. Moreover, for medium-sized firms economic incentives play an important role in guaranteeing the safeguarding of transactions, whereas big firms adopt contractual supports.
\end{abstract}

Keywords: traceability, vertical co-ordination, transaction cost economics, dairy chain

\section{Introduction}

Recent food crises in the European Union, like dioxin problems in the poultry sector and BSE in the beef sector, have enhanced consumer interest in food safety issues. In response to consequent consumer requirements, the EU has outlined new principles and set down measures for its food safety policy in order to guarantee a high level of protection for consumer health and interests.

In the context of the measures provided for the strengthening of food safety, the EU introduced traceability in the White Paper of 2000 and Regulation 178 of 2002 (defined as "general food law"). However, the growing interest in traceability is not limited just to EU policy; a number of public and private initiatives have also been taken with regard to traceability systems in various countries (Hobbs, 2004a; Souza-Monteiro and Caswell, 2004).

This paper focuses on a voluntary traceability system in Italy, established by the Italian standard organisation (UNI 10939/2001) and regulated by more complex rules than the general food law. The system allows the product itself to be traced to its origins. Our purpose here has been to analyse how the introduction of this system affects the organisation of relationships throughout the dairy chain.

Using the theoretical framework of transaction cost economics, we assess whether traceability increases the degree of vertical co-ordination and changes the level of the transaction key features, i.e. degree of asset specificity, uncertainty and frequency of transactions.

The data were collected by questionnaire, and cluster analysis was used to evaluate the attitudes, as an effect of traceability application, of the different dairy firms towards vertical co-ordination.

The paper is organised as follows: the economic issues of traceability are examined in section 2; the theoretical framework of transaction cost is presented in section 3; the survey conducted and the methodological issues are examined in section 4; the results are analysed in section 5 and the concluding remarks are set down in section 6 .

\section{Economic issues of traceability}

EU traceability is part of a framework of measures guaranteeing food safety. The guiding principle of the concept of traceability is based on the "comprehensive and integrated approach" to food safety policy introduced by the European Commission (2000) in the White Paper. As a consequence of this approach, the European food safety policy considers all sectors of the food chain, "farm to table", and assigns a primary responsibility to all the agents along the food chain.

\footnotetext{
${ }^{1}$ Corresponding author: A. Banterle. The paper is the result of the collaboration of the authors. The text is attributed as follows: section 1, 2 and 6 to A. Banterle; section 3 and 5 to S. Stranieri; section 4 to L. Baldi.
} 
In this context, traceability concerns the ability to trace and follow a food through all the stages of its production, processing and distribution, as provided by article 3 of the general food law. The main purpose of a traceability system is to reduce the probability of unsafe food, imputing specific liability to the stakeholders of the supply chains and improving information flow within the chains by means of information procedures (Meuwissen et al., 2003; Trienekens and Beulens, 2001). However, in the EU it is possible to distinguish two different traceability systems, mandatory and voluntary, which have different economic implications for firms involved in a specific supply chain.

Mandatory traceability was introduced into the EU by Reg. $1760 / 2000$ for the meat sector, and by Reg. 178/2002 for all other agri-food products. The traceability of Reg. 178/2002 can also be called "supply chain traceability", because it requires economic agents to document from whom they buy the raw materials and to whom they sell the processed products, including the relative quantities. With this kind of system it is not possible to trace the individual product unit back to the specific agricultural raw materials.

Voluntary traceability makes no reference to any EU legal framework, and different standards are outlined at the country level. In Italy the national standard organisation (UNI) has introduced standard rules for voluntary traceability (UNI 10939/2001). Recently, at the international level, the International Organisation for Standardisation introduced the general principles and basic requirements for traceability in the food chain (ISO 22005/2005), according to the UNI standard.

Voluntary traceability is a much more complex system than the mandatory one as it allows traceability at both the level of the operators involved in the supply chain (supply chain traceability) and at the level of individual firm organisation (product traceability) (Moe, 1998). With such a system it is possible for a single product to be traced back to the relative raw materials, as the firms are able to identify single raw material batches and to preserve them as separate units in the processing (Peri et al., 2004; Golan et al., 2004). In other words, the firms are able to reconstruct the complete history of a specific product, including who, and what, is involved at each stage of the food chain.

Voluntary traceability leads to positive effects on three main aspects (Vernéde et al., 2003):

- food safety, through more efficient risk management;

- food quality, thanks to the introduction of production rules and specific monitoring activities throughout the chain;

- vertical co-ordination among the economic agents. With regard to this last aspect, a firm promotes the introduction of voluntary traceability in the supply chain and involves the other economic agents of the chain. This firm thus becomes responsible for the system, managing the information flow and controls and assuming the role of leader of the chain. It is this leader firm that answers to the certification body for the correct functioning of the system. In food chains the leader firms can be retailers, processing firms and agricultural co-operatives. In our analysis the leader firms operate in the dairy industry.

In addition, the introduction of voluntary traceability in the supply chain determines an increase in bilateral dependency among the agents of the supply chain, and a reduction in the degree of transaction uncertainty as information flow is increased (Hofstede, 2002), especially with regard to the characteristics of food safety and product quality. Thus these changes lead to the reorganisation, along the supply chain, of vertical relationships that is supported by specific agreements, called supply chain agreements. These agreements establish specific production rules concerning product safety and quality, define the procedures for the management of information on product batches, and attribute specific liabilities to the participants of the supply chain.

\section{Theoretical framework}

To analyse the voluntary traceability effects on supply chain co-ordination we utilised the theoretical framework of transaction cost economics.

From the seminal work of Coase (1937), it is well known that market transactions are costly and that there are conditions under which transactions should be organised internally. The costs of transacting, called transaction costs, arise wherever there is any form of economic organisation, i.e. within a market or a vertically integrated firm, because of market failure due to information asymmetry among economic agents (Akerlof, 1970). Such information asymmetry, together with bounded rationality and opportunism of economic subjects, can lead to situations of moral hazard or adverse selection.

Transaction costs are classified in three main categories, depending on when they arise in the transaction. Information or search costs refer to costs that arise ex ante; negotiating costs take place during transactions; monitoring and enforcement costs occur ex post to a transaction after the exchange has been negotiated.

In the Williamson theory $(1985,1996)$, the governance structure of transactions depends on some key characteristics of each transaction:

- degree of asset specificity;

- degree of uncertainty surrounding the transactions;

- degree of frequency.

The first key factor, concerns the bilateral dependency of economic agents that arises when a subject invests resources to conduct transactions. There are different kinds of asset 
specificity depending on the nature of the investment, be it human asset, site asset, physical asset or dedicated asset specificity. Williamson states (1979) that the costs of governance and the need to safeguard transactions from opportunistic behaviour increase as the complexity of transaction asset specificity builds up.

The second factor depends on information asymmetry, bounded rationality of agents and the random nature of some events. It is connected principally with the probability that subjects will respect contractual obligations. Transaction costs increase when the degree of uncertainty arises. This aspect of the transaction has become very important due to new uncertainties in the food market (Hobbs, 2004b), like recent food crises and the increasing internationalisation of markets. Recently, guarantees in an institutional form to ensure food quality (Holleran et al., 1999) and closer, more competitive, vertical arrangements such as centralised modes of organisation within food supply chains (Ménard and Valceschini, 2005) are playing an important role as they decrease the uncertainty in food safety demand with regard to both the supply chain relations and the final consumers.

The third factor is connected to the time lapse between one transaction and another (Hobbs, 1996). In this analysis we did not take the level of frequency into consideration as the frequency of transactions in the dairy sector is strictly connected with product perishability. Hence, the introduction of a new governance of transactions should not lead to any variation in the frequency of transactions.

Transaction costs register an augmentation when there is an increase in the levels of frequency, uncertainty and asset specificity of the transactions. The degree of these three key transaction factors and the relative value of the information, negotiating and monitoring costs, determine a consequent governance transaction structure.

In this framework we believe that the implementation of a voluntary traceability system increases the asset specificity of transactions, decreases the relative degree of uncertainty and leads to variation in transaction costs. We can expect an increase in monitoring costs due to a growth of asset specificity after the introduction of the voluntary traceability system, and a reduction in information costs as the main aim of traceability is to increase information transparency within the food supply chains. Nevertheless, the exact quantification of the transaction costs will be extremely complex because of the many organisational costs that are not measurable.

These changes are supported by a new governance transaction structure based on supply chain agreements. Such agreements are subscribed to by all the participants along the supply chain, and are aimed at improving information transparency and trust among economic agents by means of new production rules, the introduction of a specific liability system, and verification procedures at each stage of the supply chain.

In accordance with Williamson's contracting scheme, economic actors can decide, during the formulation of the agreements and in an effort to reduce the risk of contractual infringements, whether:

- to provide economic incentives through an increase in the price of raw materials;

- to give contractual support through the introduction of, for example, specific responsibility to subjects of the traced supply chains, specific production rules, contract clauses relative to the monitoring of the activity of the operators, and sanctions applicable in the event the agreements are not respected.

Vertically integrated subjects do not follow this scheme in the formulation of supply chain agreements as they have no need to be safeguarded from opportunistic behaviour thanks to an organisation under unified ownership.

\section{Methodological issues and statistical results}

To test the changes in the vertical links after the introduction of a voluntary traceability system we conducted a survey, based on a specific questionnaire, during the spring and summer of 2005. The sample represents Italian firms certified UNI 10939 and specialised in the dairy industry. The questionnaire consists of 30 questions subdivided into three parts.

Part 1 contains questions relating to the general characteristics of the company, paying particular attention to the legal configuration, the turnover, the employees, and the level of vertical co-ordination.

Part 2 focuses on the variation in the transaction costs associated with the introduction of the voluntary system (UNI 10939) and considers especially monitoring costs. Such costs are quantified by means of questions related to the variation in both the supplier activity controls and the raw materials controls.

Part 3 is aimed at defining the changes verified in the transaction key factors following the introduction of the UNI 10939 system. The demands are related to:

- the variation of the asset specificity of transactions (changes in site, physical and human assets);

- the variation of degree of uncertainty of transactions, in terms of frequency, quantity and correctness of information.

A multiple choice format with rating scales was used to consider the answers as numerical variables (Kalton, 1983). The sample consisted of 38 firms and represents $93 \%$ of the total of firms certified UNI 10939 in the dairy sector: 
$34 \%$ of them are specialised in milk production and $66 \%$ in butter and cheese.

We applied factor analysis and then cluster analysis to identify homogeneous groups of firms in terms of their approaches to voluntary traceability. Factor analysis was carried out before cluster analysis to reduce the number of variables and find significant factors.

Factor analysis is a statistical procedure used to uncover relationships among many variables. It allows numerous intercorrelated variables to be condensed into fewer dimensions, called factors (Gorsuch, 1983). In the procedure, each variable is assumed to be a linear combination of some number of common factors and a unique factor. Principal Component Analysis (PCA) for 15 variables was used to extract the factors. This is a linear transformation of the variables that assumes those factors are able to explain all the variance in each variable. Before running PCA we calculated Bartlett's Test of Sphericity to investigate the null hypothesis that the correlation matrix between the variables is an identity matrix, and we rejected the null at 0.001 level of significance.

By SPSS we extracted 6 factors with eigenvalues greater than 1 and that together account for $76 \%$ of total variance. Orthogonal rotation (Varimax) was carried out after the initial extraction of factors. This method assumes that the six factors are not correlated and permits the redistribution of the total amount of variance over the extracted factors to make them more interpretable (see section 5 for the interpretation of factors). Factor analysis produced 6 factor score variables used for cluster analysis.

Cluster analysis is an exploratory data analysis tool to classify cases, in our case the firms, into groups, so that the degree of association is strong between cases of the same group and weak between cases of different groups (Jobson, 1992). We utilised a hierarchical approach that begins with each case describing a cluster and then combines them into more inclusive clusters until only one cluster remains. Similarity between cases was measured by Pearson's correlation.

The average linkage between groups (UPGMA) method was used to combine nearest clusters into broader groups. This technique defines the distance between two clusters as the average of the distances between all pairs of cases in the two different clusters.

The choice of the best cluster solution is subjective, nevertheless the analysis of the agglomeration schedule and dendrogram ${ }^{2}$ led to the identification of 3 clusters that seem to give the best results in terms of the following criteria: a. minimum number of firms for each cluster, i.e. a cluster must contain a sufficient number of cases in order to be representative (we assume $>4$ );

b. degree of distance between clusters, i.e. small coefficients of distance indicate the merging of fairly homogeneous clusters, whereas large coefficients show the grouping of clusters containing quite dissimilar members;

c. different characteristics among resulting clusters, i.e. the chosen groups must express distinct and significant particularities considering the analysis of the object.

\section{Results}

\section{Descriptive analysis}

The sample is composed of both big and small firms: $40 \%$ have an annual turnover lower than 10 million euros, $24 \%$ of between 10 and 25 million, 13\% between 25 and 50 million and $24 \%$ higher than 50 million. Most of the firms show a high level of vertical co-ordination. The results reveal that $58 \%$ of the firms are formed by co-operatives, the remainder by private companies. It was found that $63 \%$ of the firms are vertically integrated, $16 \%$ own some agricultural farms and $21 \%$ have no ownership of any farms. All the respondents use contracts for vertical exchanges. The motivation to introduce voluntary traceability within the dairy supply chain was mainly connected to three factors: to ensure food safety ( $61 \%$ of respondents), especially for small and medium firms; to enhance the quality characteristics of the products $(82 \%)$ and to introduce a correct liability system among the supply chain participants $(76 \%)$. The traceability system has no direct effect on the final product price in $63 \%$ of cases, is not a standard required by most of the distributors and does not improve communication among the dairy chain participants (Figure 1).

The survey underlines an increase in the degree of human, physical and site assets and a decrease in the uncertainty degree throughout the supply chain. The firms state that (Table 1):

1. a long-term business relationship is an important factor to decide which suppliers can be part of a traced supply chain (95\% of answers);

2. the substitution of certified suppliers is more difficult than for normal suppliers (50\%);

3. most of the participants involved in the certified supply chain have undergone specific training $(76 \%)$;

4. the implementation of the voluntary traceability system requires some material investment (51\%);

\footnotetext{
${ }^{2} \mathrm{~A}$ dendrogram consists of many lines connecting cases in a hierarchical tree diagram. The horizontal axis shows the 38 firms in the three clusters $(\mathrm{A}, \mathrm{B}, \mathrm{C})$; the vertical axis shows the rescaled distance measure between the two (or more) cases connected (Figure 2).
} 


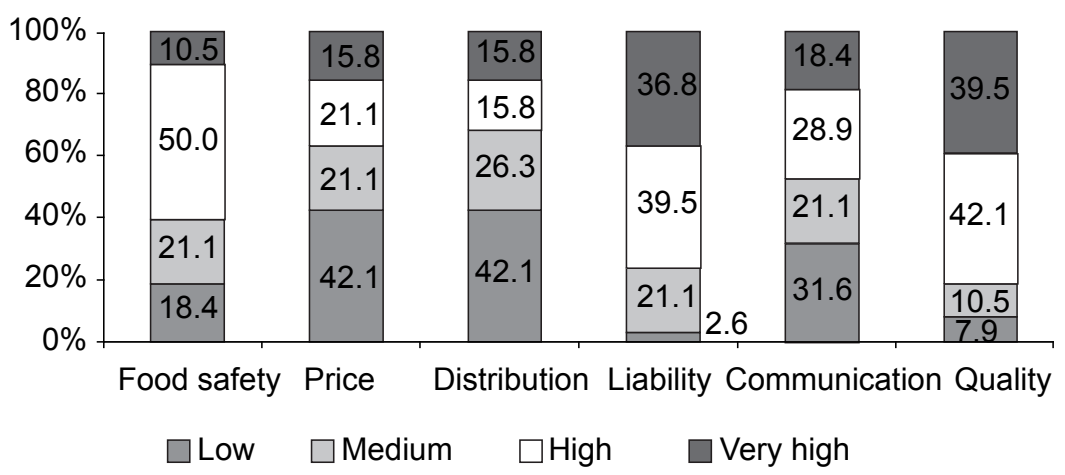

Figure 1. Level of agreement of firms regarding motivation in implementing a voluntary traceability system. Source: our survey.

Table 1. Firms' perceptions about the effects of voluntary traceability on transaction characteristics, costs and vertical co-ordination ${ }^{1}$.

Dairy firms (\%)

Low Medium High Very high Total

Firms characteristics

Turnover $^{3}$

Contracts formalisation ${ }^{2}$

Level of vertical coordination

Final product price variation ${ }^{2}$

Asset specificity variation

1. Human asset specificity variation

Long business relations with suppliers

Difficulty in substitution of suppliers

Training of suppliers

2. Physical asset specificity variation

Processing facilities variation ${ }^{2}$

3. Geographical asset specificity variation

Certified suppliers closeness

Uncertainty variation

Frequency of information flow variation

Quantity of information flow variation

Precision of information flow variation

Transaction costs variation

Monitoring costs variation

Suppliers activity controls variation

Raw materials controls variation

Proxies of vertical relationships variation

Raw materials price variation ${ }^{2}$

Liability degree variation

Production rules enforcements
39.5

5.3

21.1

71.1

$\begin{array}{rr}0.0 & 5.3 \\ 31.6 & 18.4 \\ 10.5 & 13.2\end{array}$

48.6

0.0

7.9

7.9

7.9

13.2

28.9

52.6

0.0

10.5
23.7

13.2

23.7

$94.7-100.0$

15.7

63.2

28.9

100.0

100.0
21.1

23.7

39.5

73.7

100.0

$26.3 \quad 100.0$

$36.8 \quad 100.0$

$51.4 \quad 100.0$

39.5

100.0

13.2

47.4

28.9

55.3

100.0

7.9

2.6

81.6

100.0

0.0

15.8

76.3

100.0

Source: our survey

${ }^{1}$ Questionnaire answers are scored from 1 (low) to 4 (very high); ${ }^{2}$ Answer options are yes (2=high) or no (1=low);

${ }^{3}$ low $=<10$ million euro; medium=10-25 million euro; high=25-50 million euro; very high=>50 million euro. 
5. most of the certified suppliers are located near the firm $(87 \%)$;

6. there has been an increase in frequency (84\%) and quantity (84\%) of information exchanged.

Furthermore, the interviewed firms show an increase in monitoring and enforcement costs to control supplier activities (82\% of answers) and raw materials (63\%).

\section{Factor analysis}

Factor analysis was applied to summarise the important variables that can explain the organisational changes within the dairy supply chain after the introduction of a voluntary traceability system. A rotated factor matrix was calculated to permit the interpretation of the six factors (Table 2).
The first factor explains the information expressed by the characteristics of the firms, i.e. the level of vertical coordination (the factor coefficient is equal to 0.919) and the legal configuration of firms (0.856). The second represents variables describing the variation of the transaction uncertainty degree after the introduction of the voluntary traceability system, i.e. variation in the quantity (0.898) and accuracy (0.880) of information flow.

The third factor explains production rules enforcement (0.698) and the importance of long business relations with suppliers $(0.684)$, i.e. the contractual support variation (0.698) and human asset specificity degree variation (0.684) after the introduction of the supply chain agreements. These two variables are positively correlated. According to the Williamson theory, this means that an increase in asset

Table 2. Rotated component matrix.

\begin{tabular}{|c|c|c|c|c|c|c|}
\hline & $\begin{array}{l}\text { Firms } \\
\text { characterisitcs }\end{array}$ & $\begin{array}{l}\text { Uncertainty } \\
\text { degree var. }\end{array}$ & $\begin{array}{l}\text { Contr. } \\
\text { support and } \\
\text { human asset } \\
\text { specificity } \\
\text { var. }\end{array}$ & $\begin{array}{l}\text { Monitoring } \\
\text { costs var. }\end{array}$ & $\begin{array}{l}\text { Turnover } \\
\text { and physical } \\
\text { asset var. }\end{array}$ & $\begin{array}{l}\text { Price var. } \\
\text { and var. } \\
\text { difficulty } \\
\text { suppliers } \\
\text { substitution }\end{array}$ \\
\hline \multicolumn{7}{|l|}{ Firms characteristics } \\
\hline legal shape & 0.856 & 0.123 & -0.152 & 0.083 & -0.060 & -0.229 \\
\hline turnover & -0.200 & -0.108 & 0.090 & -0.090 & 0.848 & -0.179 \\
\hline level of vertical coordination & 0.919 & -0.040 & -0.057 & -0.036 & -0.055 & -0.217 \\
\hline \multicolumn{7}{|l|}{ Asset specificity variation } \\
\hline \multicolumn{7}{|l|}{ 1. Human asset specificity variation } \\
\hline Long business relations with suppliers & 0.017 & -0.003 & 0.684 & 0.123 & 0.207 & 0.105 \\
\hline Difficulty in substitution of suppliers & -0.184 & -0.024 & -0.060 & -0.108 & -0.223 & 0.772 \\
\hline \multicolumn{7}{|l|}{ 2. Physical asset specificity variation } \\
\hline Processing facilities variation & -0.008 & 0.261 & -0.088 & 0.124 & 0.770 & 0.230 \\
\hline \multicolumn{7}{|c|}{ 3. Geographical asset specificity variation } \\
\hline Certified suppliers closeness & 0.482 & -0.175 & 0.432 & 0.097 & -0.288 & 0.132 \\
\hline \multicolumn{7}{|l|}{ Uncertainty variation } \\
\hline Frequency of information flow variation & -0.110 & 0.271 & 0.777 & -0.055 & -0.018 & 0.048 \\
\hline Quantity of information flow variation & 0.144 & 0.898 & 0.079 & -0.071 & 0.048 & 0.030 \\
\hline Precision of information flow variation & -0.129 & 0.880 & -0.062 & 0.236 & 0.046 & -0.023 \\
\hline \multicolumn{7}{|l|}{ Transaction costs variation } \\
\hline \multicolumn{7}{|l|}{ Monitoring costs variation } \\
\hline Suppliers activity controls variation & -0.005 & 0.166 & -0.061 & 0.900 & 0.212 & 0.000 \\
\hline Raw materials controls variation & 0.410 & 0.132 & -0.016 & 0.627 & -0.272 & 0.173 \\
\hline \multicolumn{7}{|l|}{ Proxies of vertical coordination variation } \\
\hline Raw materials price variation & -0.179 & 0.048 & 0.188 & 0.059 & 0.291 & 0.786 \\
\hline Liability degree variation & -0.084 & -0.147 & 0.398 & 0.742 & -0.082 & -0.242 \\
\hline Production rules enforcements & -0.110 & -0.390 & 0.698 & 0.052 & -0.243 & -0.127 \\
\hline
\end{tabular}

Source: our survey. 
specificity determines a positive variation of production and contractual conditions to safeguard transactions from the opportunistic behaviour of economic participants. The fourth factor is mainly connected to variables explaining variation in monitoring costs, i.e. supplier activity (0.900) and raw material controls $(0.627)$. In this factor also, the variable explaining the liability degree variation plays an important role (0.742). The fifth factor explains firm turnover (0.848) and physical asset variation (0.770). The last one represents contractual economic incentives provided by firms (0.786) and the variation in the difficulty of supplier substitution after the introduction of voluntary traceability (0.772).

\section{Cluster analysis}

Cluster analysis (CA) applied to the components described above reveals the presence of three groups of firms, as the dendrogram shows in Figure 2. These groups differ from each other in terms of voluntary traceability effects on vertical co-ordination.

To facilitate the interpretation of the clusters, we calculated the average of factorial coefficients of the firms in each cluster for each component.

In agreement with the Williamson perspective, vertically integrated firms do not assist in any increase in the degree of asset specificity or variation in transaction governance, whereas firms that mainly use contracts to conduct transactions show variations in the transaction key characteristics and contractual safeguards after the introduction of the voluntary system. It should also be noted that each cluster contains firms that chose different organisational solutions to promote continuity and safeguard transactions (Figure 3 ).

The first group, which is explained by the factor of firm characteristics (0.683), consists of sixteen firms, most of which are small co-operatives with an annual turnover lower than 10 million euros and an average number of 30 employees. These firms do not show any increase in human asset (-0.27) and physical asset specificity (-0.24) but do have a greater quantity and precision of information exchanged (0.49). In this case the firms do not report any increase in vertical co-ordination as transactions are already safeguarded internally and there is no need for a strengthening of economic incentives or contractual supports.

The second group is composed of 12 firms, most of which have more than 70 employees and an annual turnover of between 10 and 25 million euros. These firms reveal little variation in bilateral dependency because of the increase in human asset specificity and the reduction in the transaction uncertainty level (0.30). According to Williamson, the increase in information transparency counteracts the opportunistic behaviour of economic agents, and any increase in bilateral dependency is safeguarded by economic incentives to certified suppliers. Thus, in this case, the increase in the price $(0.60)$ of raw materials is sufficient to

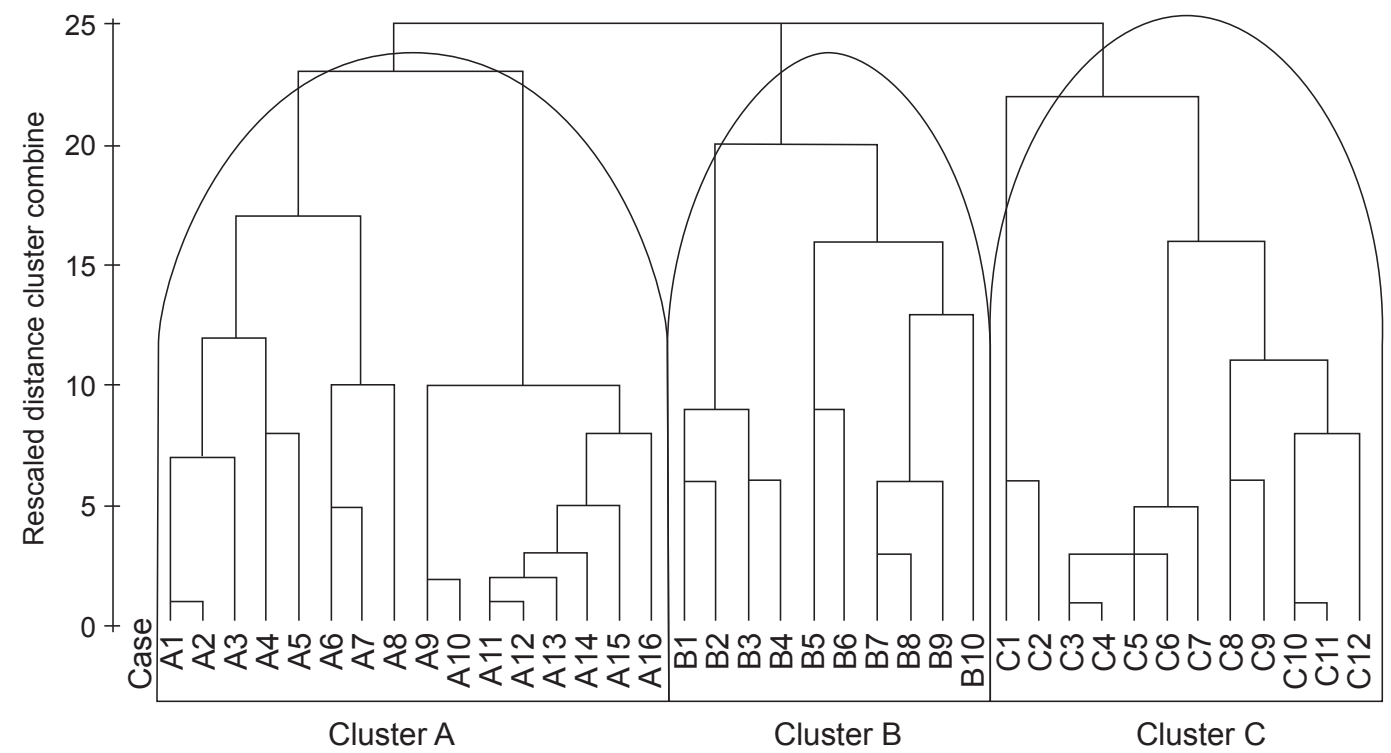

Figure 2. Dendrogram using average linkage between groups method in which are shown 3 clusters of firms (A, B, C). Source: our survey. 


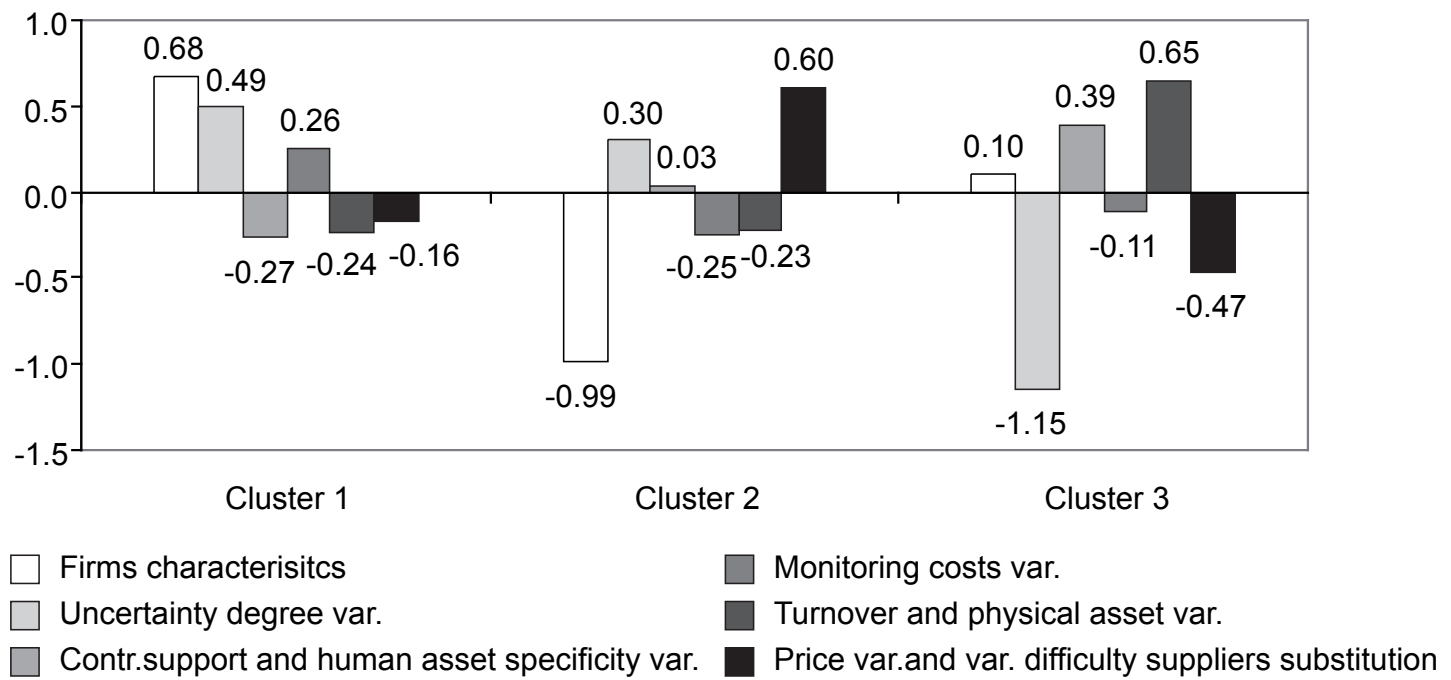

Figure 3. Average of factorial coefficients in each cluster. Source: our survey.

safeguard transactions and to ensure the correct execution of transactions.

The last cluster is represented by 10 big private firms with more than 100 employees and an annual turnover higher than 100 million euros. Most of these firms own some farms (the coefficient describing the level of vertical co-ordination is 0.10 ) and are characterised by contracts for exchanges. These firms reveal a higher increase in human and physical (0.65) asset specificity than those belonging to the second cluster. The respondents in this cluster did not show any reduction in information asymmetry $(-1.15)$, because most of them implement the system on their farms and already use other certification systems. The positive variation of bilateral dependency connected with the traceability system is safeguarded by new contractual supports, like liability variations and production rules enforcement. The factor explaining this information has a factorial coefficient of 0.39 .

Cluster analysis reveals that any variation in governance of the supply chain brought about by the introduction of voluntary traceability depends on several variables related to the general characteristics of the firms and the variations of transaction factors. Indeed, such variations lead to changes in the transaction costs.

With regard to the firms' characteristics, the size and the level of vertical co-ordination influence the reorganisation of the exchange. The first cluster consists of small, vertically integrated companies, the second of mediumsized companies and the third of big companies. The second and third clusters group together companies that use written agreements to regulate the exchanges, but they differ in the contractual conditions aimed at guaranteeing the transparency of exchanged information (in the second cluster the firms use economic incentives, in the third contractual safeguards).

With regard to the transaction factors, the variation in the uncertainty level and asset specificity, verified by the introduction of voluntary traceability, influences the content of supply chain agreements for traced agricultural raw material. If there is an increase in only informative transparency, the level of vertical co-ordination remains more or less the same. If there is a reduction in the level of uncertainty and a modest increase in asset specificity, economic incentives are established for the traced suppliers. If the asset specificity increases, the economic operators introduce greater contractual safeguards to guarantee the correct application of the traceability system.

Furthermore, the transaction governance based on supply chain agreements leads to variation in monitoring costs. If the content of these agreements remains the same, these costs increase. If, instead, the supply chain agreements introduce economic incentives or additional safeguards, the monitoring costs decrease thanks to an augmentation of contractual safeguards and a reduction of possible opportunistic behaviour of the economic subjects.

\section{Concluding remarks}

The analysis carried out reveals notable differences, in the European Union, between voluntary traceability and mandatory traceability. This latter is based, as set down by the general food law, on adopting an information management procedure for the exchange of goods along the supply chain, but it does not allow the association of 
this information with the individual product. Mandatory traceability is mainly aimed at improving the level of product food safety, increasing the amount of information in the supply chain and allocating specific responsibilities to the agents of the supply chain. Therefore such a system will not result in a reorganisation of the vertical relations in the supply chains, it can only lead to limited changes.

Voluntary traceability is based on two fundamental points:

- management of the raw materials and products by separate batches, both for exchange among agents of the supply chain and in the transformation processes within the company;

- management of information, so that each individual batch has its own associated information.

This means that it is possible to reconstruct the route of a single product unit within the supply chain. Also with this system there is an increase in the information and the attribution of specific liabilities to the supply chain agents, to a greater degree than with mandatory traceability due to the management of separate batches.

The empirical analysis of the Italian dairy chain has revealed that the firms' purposes for the introduction of voluntary traceability are multiple, but two motives are particularly significant:

- to increase food safety and improve risk management so that, in the event of non-conformity, it is possible to withdraw only the single batch from the market;

- to improve food quality standards through the introduction of production rules and controls for the agents of the supply chain.

Voluntary traceability requires a reorganisation of vertical relations based on supply chain agreements, and this represents a new mechanism for transaction governance.

From the transaction cost perspective, the survey showed increased bilateral dependency of the economic agents as a consequence of the rise in the degree of human, physical and site assets. At the same time, growth was observed in the frequency and quantity of information exchanged, underlining, throughout the supply chain, a decrease in the degree of uncertainty. Moreover, the interviewed firms revealed an increase in monitoring costs for the checking of supplier activities and raw materials.

With regard to the prices of the traced products, the empirical analysis highlighted that $71 \%$ of the firms did not claim any increase in the price of the final product, while the remaining $29 \%$ obtained premium prices because of the applied voluntary traceability. Instead, $47 \%$ of the processing firms reported that the price of the traced raw materials, the milk they bought, increased.
Cluster analysis underlined three different vertical organisational arrangements connected to the introduction of voluntary traceability, depending on firm size and the level of vertical co-ordination. The first group consists of small co-operatives with no variation in the transaction key factors, though there is higher information flow. The second cluster is composed of medium-sized firms that show a reduction in the uncertainty level of transactions; here, economic incentives play an important role in guaranteeing the safeguarding of transactions. Finally, the third group is represented by big firms characterised by a high growth of human and physical asset specificity. In this case contractual supports are provided to safeguard the transactions.

Our empirical analysis has highlighted different positive aspects for firms operating in the Italian dairy chain as a consequence of the voluntary traceability system. However, few dairy firms have adopted voluntary traceability, and the reason for this could be the difficulties encountered in implementing the system and in gaining consumer recognition.

In this sense, it is interesting to mention the benefits and the costs of voluntary traceability at the level of company management. With regard to the benefits, in addition to the advantages related to food safety and product quality of which we have already spoken, it can be underlined that the adoption of voluntary traceability can create, for the leader firm, a competitive advantage with various benefits depending on the choices made by the company, such as premium prices for traced products (29\% of firms in our analysis); improved brand image and an increase in the market share; greater bargaining power with regard to others in the supply chain; greater presence in foreign markets where there are protocols calling for these traceability systems. Furthermore, for agricultural farms the advantages can be related to both price and greater guarantees for the sale of products consequent to the growth of bilateral dependency among supply chain agents.

With regard to costs, those occurring in the initial phase of the system implementation concern mainly the costs of adapting production processes for the management of separate batches; information system costs; the costs of certification, and the costs of training of personnel. In the management phase of the system the main costs are related to the cost of labour and product promotion.

Therefore the adoption of voluntary traceability represents a strategic choice to be made by the company and has to be evaluated at the level of the specific company management. Communicating the characteristics of voluntary traceability, in terms of safety and quality guarantees of the food product, to the end consumer remains a critical factor. 


\section{References}

Akerlof, G.A., 1970. The market for "lemons": quality uncertainty and the market mechanism. The Quarterly Journal of Economics 84(3), 488-500.

Coase, R.H., 1937. The nature of the firm. Economica. New Series 4(16), 386-405.

European Commission, 2000. White Paper on Food Safety. COM(1999) 719, Brussels.

Golan, E., B. Krissoff, F. Kuchler, L. Calvin, K. Nelson and G. Price, 2004. Traceability in the US food supply: Economic theory and industry studies. Agricultural economic report 830, USDA.

Gorsuch, R.L., 1983. Factor Analysis. Hillsdale, NJ, Lawrence Erlbaum.

Hobbs, J.E., 1996. A transaction cost approach to supply chain management. Supply Chain Management 1(2), 15-27.

Hobbs, J.E., 2004a. Information Asymmetry and the Role of Traceability Systems. Agribusiness 20(4), 397-415.

Hobbs, J.E., 2004b. Markets in metamorphosis: the rise and fall of Policy Institutions. In G. Van Huylenbroeck, W. Verbeke and L. Lawers (editors), Role of Institutions in Rural Policies and Agricultural Markets. Elsevier, The Netherlands, pp. 199-210.

Hofstede, G.J., 2002. Transparency in Netchains. Review paper for Klict 16, KLICT3949.

Holleran, E., M.E. Bredhal and L. Zaibet, 1999. Private incentives for adopting food safety and quality assurance. Food Policy 24, 669-683.

Jobson, J.D., 1992. Applied Multivariate Data Analysis (Vol. 2). Springer-Verlag, New York.

Kalton, G., 1983. Introduction to Survey Sampling. Sage Publications, Beverly Hills.

Ménard, C. and E. Valceschini, 2005. New institutions for governing the agri-food industry. European Review of Agricultural Economics 32(3), 421-440.

Meuwissen, M.P.M., A.G.J Velthuis, H. Hogeveen and R.B.M. Huirne, 2003. Technical and economic considerations about traceability and certification in livestock production chains. In A.G.J. Velthuis, L.J. Unnevehr, H. Hogeveen and R.B.M. Huirne (editors), New approaches to food safety economics. Kluwer Academic Publishers, The Netherlands, pp. 41-54.

Moe, T., 1998. Perspectives on traceability in food manufacture. Trends in Food Science \& Technology 9, 211-214.

Peri, C., L. Pellegrini, G. Gay and N. Fabbri, 2004. Importanza della tracciabilità, in una logica di forte identità delle produzioni agroalimentari della Lombardia, e conseguenti strategie di marketing da adottare relativamente alle principali filiere produttive lombarde. Irer, Milano.

Souza-Monteiro, D.M. and J.A. Caswell, 2004. The Economics of Implementing Traceability in Beef Supply Chains: Trends in Major Producing and Trading Countries. Department of Resource Economics, University of Massachusetts Amherst.
Trienekens, J. and A. Beulens, 2001. The implications of EU food safety legislation and consumer demands on supply chain information systems. Working paper of IAMA Symposium 2001, Wageningen University, Dept. Management studies.

Velthuis, A.G.J., L.J. Unnevehr, H. Hogeveen and R.B.M. Huirne (editors), 2003. New approaches to food safety economics. Kluwer Academic Publishers, The Netherlands.

Vernéde, R., F. Verdenius and J. Broeze, 2003. Traceability in food processing chains. State of the art and future developments. Klict position paper, Agrotechnology \& Food Innovations bv, Wageningen.

Williamson, O.E., 1979. Transaction cost economics: the governance of contractual relations. Journal of Law and Economics 22, 233262.

Williamson, O.E., 1985. The economic institution of capitalism. Free Press, New York.

Williamson, O.E., 1996. The mechanism of governance. Oxford University Press, New York.

Williamson, O.E., 2004. Transaction cost economics and agriculture: an excursion. In G. Van Huylenbroeck, W. Verbeke and L. Lawers (editors), Role of Institutions in Rural Policies and Agricultural Markets. Elsevier, The Netherlands, pp. 19-37. 